\title{
Quantum chaos analysis of the ideal interchange spectrum in a stellarator
}

\author{
R. L. DEW A R, ${ }^{1}$ C. N ÜH R E B E R G ${ }^{2}$ and T. T A T S U N O \\ ${ }^{1}$ Department of Theoretical Physics and Plasma Research Laboratory, \\ Research School of Physical Sciences and Engineering, The Australian \\ National University, Canberra ACT 0200, Australia \\ (robert.dewar@anu.edu.au) \\ ${ }^{2}$ Max-Planck-Institut für Plasmaphysik, Teilinstitut Greifswald IPP-EURATOM \\ Association, D-17489 Greifswald, Germany \\ ${ }^{3}$ Center For Scientific Computation and Mathematical Modeling, University of Maryland, \\ College Park, MD 20742-3289, USA
}

(Received 17 August 2005 and accepted 2 February 2006)

\begin{abstract}
The eigenmode spectrum is a fundamental starting point for the analysis of plasma stability and the onset of turbulence. Quantum chaos theory provides tools for characterizing the spectrum statistically, from the regular spectrum of the separable case (integrable semiclassical dynamics) to that where the semiclassical ray dynamics is so chaotic that no simple classification of the individual eigenvalues is possible (quantum chaos). Using the CAS3D code, a data set of several hundred growth-rate eigenvalues has been calculated for a Mercier-unstable threedimensional stellarator equilibrium with a rather flat, non-monotonic rotational transform profile. Statistical analysis of eigenvalue spacings for individual mode families shows evidence of quantum chaos, strongest for the $N=0$ family, but to test this we compare it with the distribution of eigenvalue spacings in a similar separable case -ideal interchange modes in a Suydam-unstable plasma cylinderusing a similar rotational transform profile to the stellarator case. The statistics in the cylindrical model appear Poissonian, as expected for generic integrable systems and in clear contrast to the three-dimensional stellarator results.
\end{abstract}

\section{Introduction}

Numerical matrix eigenvalue programs, such as the three-dimensional TERPSICHORE [1] and CAS3D [2] codes, are routinely used to assess the ideal magnetohydrodynamic (MHD) stability of candidate configurations for proposed fusionrelevant experiments with complicated geometries. An example is in the design of the five-fold-symmetric Wendelstein 7-X (W7-X) stellarator, currently under construction, where CAS3D was used [3] to study a number of different cases.

The three-dimensional nature of stellarator equilibria breaks all continuous symmetries, coupling both poloidal $(m)$ and toroidal $(n)$ Fourier harmonics. This precludes separation of variables and simple classification of the eigenvalues. As a consequence, the nature of the spectrum is still imperfectly understood, making both the physical interpretation of stability calculations and the rigorous numerical analysis of convergence difficult. 


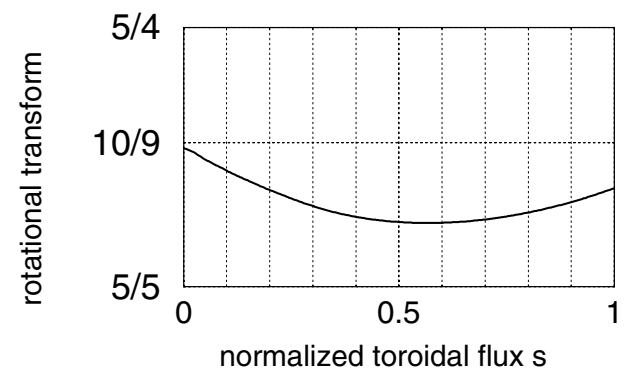

(a)

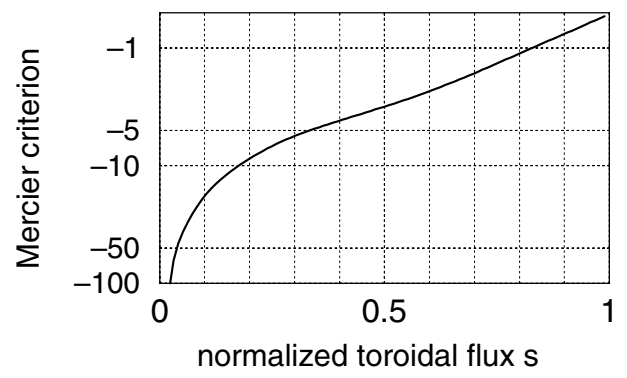

(b)

Figure 1. (a) The rotational transform $t=t(s)$ profile versus normalized toroidal flux $s\left(s \propto r^{2}\right.$ near the magnetic axis). (b) A measure of the Mercier stability versus normalized toroidal flux for our unstable W7-X variant equilibrium. (A negative value indicates instability.)

The optimized configurations used in designing W7-X are MHD stable, but in the present paper we are concerned with a variant, unstable equilibrium from this study, a high-mirror-ratio, high-rotational transform case. Its less-pronounced shaping makes it quite unstable, thus allowing us to perform a numerical experiment designed to explore some fundamental properties of the MHD spectrum in stellarators without the added complication of the stable Alfvén spectrum.

The eigenvalues, $\omega^{2} \equiv-\gamma^{2}$, are real due to the self-adjointness of the force and kinetic energy operators in ideal MHD, linearized about a static equilibrium. This is analogous to the Hermitian nature of quantum mechanics. Thus, we study the W7$\mathrm{X}$ Mercier (interchange)-unstable case mentioned above using statistical techniques from the theory of quantum chaos (see, e.g., [4]).

As a non-chaotic reference case we also study, following [5], the interchange unstable spectrum in an effectively cylindrical model of a stellarator. In the cylindrical case the eigenvalue problem is separable into three one-dimensional eigenvalue problems, with radial, poloidal, and toroidal (axial) quantum numbers $l, m$, and $n$, respectively. If the spectrum falls within the generic quantum chaos theory universality class for integrable, non-chaotic systems, then the probability distribution function for the separation of neighboring eigenvalues is a Poisson distribution [4].

In [5] we found that the spectrum for the most unstable ideal MHD interchange modes in a cylinder, those with radial mode number $l=0$, gave highly nonPoissonian statistics (when we used a sharp truncation in $m$ and $n$ ). However, it is likely that the statistics of the full spectrum, including the many weakly unstable modes with $l>0$ near the interchange accumulation point at the origin, is closer to Poisson statistics because of the occurrence of many accidental degeneracies.

In the present paper we compare the results of the quantum chaos analysis of the W7-X $N=1$ mode family [2] with eigenvalues from a cylindrical model with similar rotational transform profile.

\section{Numerical results}

The magnetic field lines of the $\mathrm{W} 7-\mathrm{X}$ variant equilibrium studied are taken to lie on nested toroidal flux surfaces labelled by $s \in[0,1]$, the enclosed toroidal magnetic flux divided by the toroidal flux enclosed by the plasma boundary. The magnetic field is characterized on each flux surface by its winding number $t(s)$. As seen in Fig. 1, the rotational transform profile is non-monotonic and has low shear 


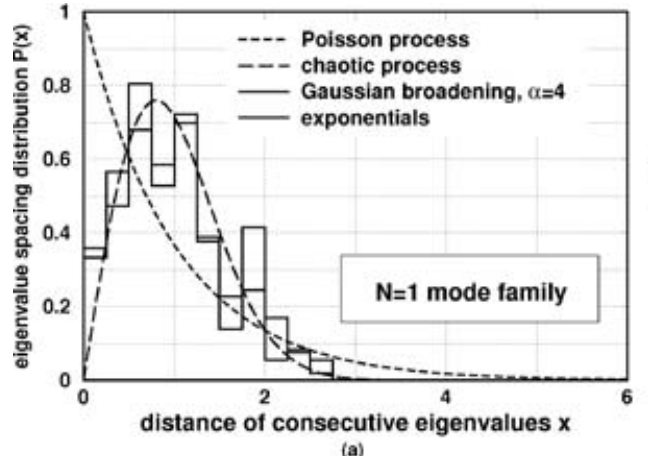

(a)

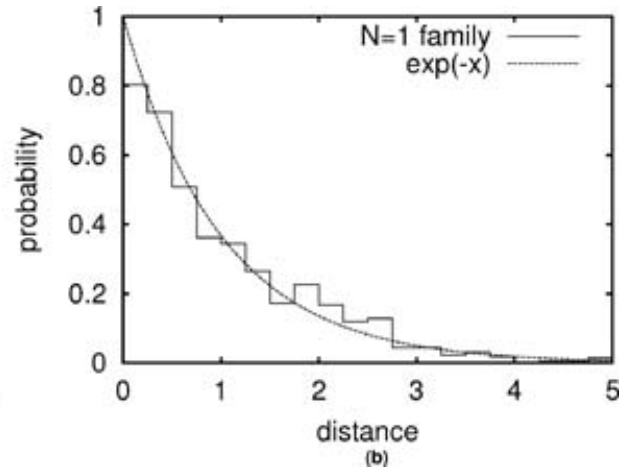

Figure 2. (a) Unfolded eigenvalue spacing distributions for $\mathrm{W} 7-\mathrm{X}$ mode family $N=1$, calculated using two different unfolding methods. (b) The same statistic from the cylindrical comparison case.

$\left(t_{\mathrm{axis}}=1.1066, t_{\min }=1.0491, t_{\mathrm{edge}}=1.0754\right)$ so it is close to, but greater than, unity over the whole plasma. As also seen from Fig. 1, the equilibrium is interchange unstable because the Mercier stability criterion is violated over the whole plasma.

In the CAS3D code, the radial dependence of the perturbation functions is treated by a hybrid finite-element approach, using a linear interpolation for the normal displacement and piecewise constant interpolations for the scalar components that describe the MHD displacement within the magnetic surfaces. Fixed boundary conditions were used with incompressible perturbations and a non-physical kinetic energy normalization. (As the eigenvalues are renormalized - 'unfolded' in quantum chaos language - to give an average separation of unity, the precise normalization used in the code is not important for the statistics.) In the calculations discussed here, 301 radial grid points were used, but a run with 451 grid points gave the same qualitative results. For the plot in Fig. 2, 99 Fourier harmonics were used, distributed with half-bandwidth 5 about $n / m=1$, where $n$ and $m$ are the toroidal and poloidal Fourier indices, respectively, with $n_{\max }=21$. There were 214 unstable eigenvalues found in this case, and all were used in the statistical analysis.

Owing to the five-fold symmetry of the equilibrium, any toroidal Fourier harmonic $n$ in an eigenfunction is coupled to toroidal harmonics $n \pm 5$. With the poloidal harmonics chosen to be positive, $m \geq 0$, there are just three uncoupled mode families $N=0,1,2$ (cf. [2]).

After unfolding the eigenvalues so that their average separation is unity, we characterize the statistics of the ensembles of eigenvalues within the $N=1$ mode family using a standard statistic from quantum chaos theory $[4,6]$, the probability distribution function, $P(x)$, for the eigenvalue separation $x$, shown in Fig. 2 .

As seen from Fig. 2(a), the eigenvalue spacing distribution function for the $\mathrm{W} 7-\mathrm{X}$ case is closer to the form found in generic chaotic systems [4] than to the Poisson distribution for separable systems, giving support for the quantum chaos prediction of [7]. The same is true for the other two mode families. (Although the $N=2$ case appeared to deviate significantly from the Wigner-conjecture distribution using 301 grid points, when the number was increased to 451 grid points, giving 402 eigenvalues, the distribution became much closer to quantum chaotic.)

To test the sensitivity to the precise method of unfolding chosen, we calculated the statistics using two different methods. They are the Gaussian unfolding method [6] and a fit with exponentials. The results, shown in Fig. 2(b) are seen to be 
consistent to within statistical error, indicating little sensitivity to the unfolding method.

The calculations for the cylindrical reference case were done with a shooting code, so the radial resolution was essentially infinite. To keep the set of eigenvalues finite, and to model the finite radial resolution of CAS3D, only at most 101 radial eigenmodes were kept for each unstable $(m, n)$, giving a dataset of 907 eigenvalues, 200 of which are non-resonant. Figure $2(b)$ shows the statistics to be close to Poissonian.

\section{Conclusion}

Although space has precluded us from showing the results for the $N=0$ and 1 mode families, in all three families the spacing distributions in the $\mathrm{W} 7-\mathrm{X}$ case are close to that expected from quantum chaos theory, while those for the cylindrical reference case are close to the Poisson distribution. We therefore conclude that interchange instabilities in the $\mathrm{W} 7-\mathrm{X}$ helias are strongly three-dimensional.

This contrasts with earlier work on the 10-field-period Large Helical Device heliotron/torsatron $[8,9]$ where the interchange spectrum was found to be rather regular, which was interpreted as being due to the averaging effect of the interchange eigenfunctions being extended over a large number of field periods.

\section{Acknowledgement}

RLD acknowledges support by the Australian Research Council.

\section{References}

[1] Anderson, D. V., Cooper, W. A., Gruber, R., Merazzi, S. and Schwenn, U. 1990 Int. J. Supercomput. Appl. 4, 34 .

[2] Schwab, C. 1993 Ideal magnetohydrodynamics: global mode analysis of threedimensional plasma configurations. Phys. Fluids B 5, 3195-3206.

[3] Nührenberg, C. 1996 Global ideal magnetohydrodynamic stability analysis for the configurational space of Wendelstein 7-X. Phys. Plasmas 3, 2401-2410.

[4] Haake, F. 2001 In: Quantum Signatures of Chaos, 2nd edn. Berlin: Springer.

[5] Dewar, R. L., Tatsuno, T., Yoshida, Z., Nührenberg, C. and McMillan, B. F. 2004 Statistical characterization of the interchange-instability spectrum of a separable idealmagnetohydrodynamic model system. Phys. Rev. E 70, 066409.

[6] Bruus, H. and d'Auriac, J.-C. A. 1997 Energy level statistics of the two-dimensional Hubbard model at low filling, Phys. Rev. B 55, 9142-9159.

[7] Dewar, R. L., Cuthbert, P. and Ball, R. 2001 Strong 'quantum' chaos in the global ballooning mode spectrum of three-dimensional plasmas. Phys. Rev. Lett. 86, 2321.

[8] Cooper, W. A., Singleton, D. B. and Dewar, R. L. 1996 Spectrum of ballooning instabilities in a stellarator. Phys. Plasmas 3, 275-280 (Erratum: 1996 Phys. Plasmas 3, 3520).

[9] Cuthbert, P., Lewandowski, J. L. V., Gardner, H. J., Persson, M., Singleton, D. B., Dewar, R. L., Nakajima, N. and Cooper, W. A. 1998 Toroidally localized and nonlocalized ballooning instabilities in a stellarator. Phys. Plasmas 5, 2921-2931. 